CUBO A Mathematical Journal

Vol.12, Noㅡ, (43-52). June 2010

\title{
On subsets of ideal topological spaces
}

\author{
V. RENUKADEVI \\ Department of Mathematics, \\ ANJA College, \\ Sivakasi-626 124, Tamil Nadu, India. \\ email: renu_siva2003@yahoo.com
}

\begin{abstract}
We define some new collection of sets in ideal topological spaces and characterize them in terms of sets already defined. Also, we give a decomposition theorem for $\alpha$ - $\mathcal{I}$-open sets and open sets. At the end, we discuss the property of some collection of subsets in $\star$-extremally disconnected spaces.
\end{abstract}

\section{RESUMEN}

Definimos una nueva colección de conjuntos en espacios topológicos ideales y caracterizamos estos en términos de conjuntos ya definidos. También damos un teorema de descomposición para $\alpha-\mathcal{I}$ - abiertos y conjuntos abiertos. Finalmente discutimos la probabilidad de algunas colecciones de subconjuntos en espacios disconexos $\star-$ extremos.

Key words and phrases: $\star-$ extremally disconnected spaces, $t-\mathcal{I}$-set, $\alpha-\mathcal{I}$-open set, pre-I-open set, semi-I-open set, semi ${ }^{\star}-\mathcal{I}$-open, semipre ${ }^{\star}-\mathcal{I}$-open, $\mathcal{C}_{\mathcal{I}}-$ set, $\mathcal{B}_{\mathcal{I}}-$ set, $\mathcal{B}_{1 \mathcal{I}}-$ set, $\mathcal{B}_{2 \mathcal{I}}-$ set, $\mathcal{B}_{3 \mathcal{I}}-$ set, $\delta-\mathcal{I}$-open, $R \mathcal{I}$-open, $\mathcal{I}$-locally closed set, weakly $\mathcal{I}$-locally closed set, $\mathcal{A}_{\mathcal{I} R}-$ set, $\mathcal{D}_{\mathcal{I}}-$ set.

2000 AMS subject Classification: Primary: 54 A 05, 54 A 10 


\section{Introduction}

By a space, we always mean a topological space $(X, \tau)$ with no separation properties assumed. If $A \subset X, \operatorname{cl}(A)$ and $\operatorname{int}(A)$ will, respectively, denote the closure and interior of $A$ in $(X, \tau)$. An ideal $\mathcal{I}$ on a topological space $(X, \tau)$ is a nonempty collection of subsets of $\mathrm{X}$ which satisfies (i) $A \in \mathcal{I}$ and $B \subset A$ implies $B \in \mathcal{I}$ and (ii) $A \in \mathcal{I}$ and $B \in \mathcal{I}$ implies $A \cup B \in \mathcal{I}$. Given a topological space $(X, \tau)$ with an ideal $\mathcal{I}$ on $\mathrm{X}$ and if $\wp(X)$ is the set of all subsets of $X$, a set operator $(.)^{\star}: \wp(X) \rightarrow \wp(X)$, called a local function [14] of $A$ with respect to $\tau$ and $\mathcal{I}$, is defined as follows: for $A \subset X, A^{\star}(\mathcal{I}, \tau)=\{x \in$ $X \mid U \cap A \notin \mathcal{I}$ for every $U \in \tau(x)\}$ where $\tau(x)=\{U \in \tau \mid x \in U\}$. We will make use of the basic facts concerning the local function [11, Theorem 2.3] without mentioning it explicitly. A Kuratowski closure operator $c l^{\star}()$ for a topology $\tau^{*}(\mathcal{I}, \tau)$, called the $\star-$ topology, finer than $\tau$ is defined by $c l^{\star}(A)=A \cup A^{\star}(\mathcal{I}, \tau)[16]$. When there is no chance for confusion, we will simply write $A^{\star}$ for $A^{\star}(\mathcal{I}, \tau)$ and $\tau^{\star}$ or $\tau^{\star}(\mathcal{I})$ for $\tau^{\star}(\mathcal{I}, \tau)$. int $t^{\star}(A)$ will denote the interior of $A$ in $\left(X, \tau^{\star}\right)$. If $\mathcal{I}$ is an ideal on $X$, then $(X, \tau, \mathcal{I})$ is called an ideal space. A subset $A$ of an ideal space $(X, \tau, \mathcal{I})$ is $\tau^{\star}-$ closed or $\star-$ closed $[11]($ resp. $\star-p e r f e c t[10])$ if $A^{\star} \subset A\left(\operatorname{resp} . A=A^{\star}\right)$. A subset $A$ of an ideal space $(X, \tau, \mathcal{I})$ is said to be a $t-\mathcal{I}-\operatorname{set}[8]$ if $\operatorname{int}(A)=\operatorname{int}\left(c l^{\star}(A)\right)$. A subset $A$ of an ideal space $(X, \tau, \mathcal{I})$ is said to be $\delta-\mathcal{I}-$ open $[2]($ resp. $\alpha-\mathcal{I}-$ open [8],pre $-\mathcal{I}-$ open $[6]$, semi $-\mathcal{I}-$ open [8], strong $\beta-\mathcal{I}-$ open $[9])$ if $\operatorname{int}\left(c l^{\star}(A)\right) \subset c l^{\star}(\operatorname{int}(A))\left(\operatorname{resp} . \quad A \subset \operatorname{int}\left(c l^{\star}(\operatorname{int}(A))\right), A \subset \operatorname{int}\left(c l^{\star}(A)\right), A \subset \operatorname{cl} l^{\star}(\operatorname{int}(A), A \subset\right.$ $c l^{\star}\left(\operatorname{int}\left(c l^{\star}(A)\right)\right)$. We will denote the family of all $\delta-\mathcal{I}$-open (resp. $\alpha-\mathcal{I}$-open, pre-I-open, semi-I-open, strong $\beta-\mathcal{I}$-open) sets by $\delta \mathcal{I} O(X)($ resp. $\alpha \mathcal{I} O(X), P \mathcal{I} O(X), S \mathcal{I} O(X), s \beta \mathcal{I} O(X))$. The largest pre $\mathcal{I}$-open set contained in $A$ is called the pre-I-interior of $A$ and is denoted by $p \mathcal{I}$ int $(A)$. For any subset $A$ of an ideal space $(X, \tau, \mathcal{I}), \operatorname{pIint}(A)=A \cap \operatorname{int}\left(c l^{\star}(A)\right)\left[15, \operatorname{Lemma}_{1.5}\right]$.

\section{Subsets of Ideal Topological Spaces}

Let $(X, \tau, \mathcal{I})$ be an ideal space. A subset $A$ of $X$ is said to be a semi $i^{\star} \mathcal{I}$-open set [7] if $A \subset \operatorname{cl}\left(\operatorname{int}^{\star}(A)\right)$. A subset $A$ of $X$ is said to be a semi $i^{\star}-\mathcal{I}$-closed set [7] if its complement is a semi $i^{\star}-\mathcal{I}$-open set. Clearly, $A$ is semi $i^{\star}-\mathcal{I}$-closed if and only if $\operatorname{int}\left(c l^{\star}(A)\right) \subset A$ if and only if $\operatorname{int}\left(c l^{\star}(A)\right)=\operatorname{int}(A)$ and so semi $i^{\star}-\mathcal{I}$-closed sets are nothing but $t-\mathcal{I}$-sets. $A$ is said to be a semipre $^{\star}-\mathcal{I}$-closed set if $\operatorname{int}\left(c^{\star}(\operatorname{int}(A))\right) \subset A$. Clearly, $A$ is said to be a semipre $e^{\star}-\mathcal{I}-$ closed if and only if $\operatorname{int}\left(c l^{\star}(\operatorname{int}(A))\right)=\operatorname{int}(A)$ if and only if $A$ is $\alpha^{\star}-\mathcal{I}$-set [8]. Clearly, $X$ is both semi $i^{\star}$ $\mathcal{I}$-closed and semipre $e^{\star}-\mathcal{I}$-closed. The smallest semi $i^{\star}-\mathcal{I}$-closed (resp. semipre $e^{\star}-\mathcal{I}-$ closed) set containing is called the semi $i^{\star}-\mathcal{I}-$ closure (resp.semipre $e^{\star}-\mathcal{I}-$ closure) of $A$ and is denoted by $S \mathcal{I} c l(A)(\operatorname{resp} . s p \mathcal{I} c l(A))$. A subset $A$ of an ideal space $(X, \tau, \mathcal{I})$ is said to be a $\mathcal{B}_{\mathcal{I}}-\operatorname{set}[8]$ if $A=U \cap V$ where $U$ is open and $V$ is a $t-\mathcal{I}$-set. The easy proof of the following Theorem 2.1 is omitted which says that the arbitrary intersection of semi ${ }^{\star}-\mathcal{I}$-closed (resp. semipre $-\mathcal{I}$-closed) set is a semi $^{\star}-\mathcal{I}$-closed (resp. semipre ${ }^{\star}-\mathcal{I}$-closed) set.

Theorem 2.1. Let $(X, \tau, \mathcal{I})$ be an ideal space and $A \subset X$. If $\left\{A_{\alpha} \mid \alpha \in \triangle\right\}$ is a family of semi $i^{\star}$ $\mathcal{I}$-closed (resp. semipre $e^{\star}-\mathcal{I}$-closed) sets, then $\cap A_{\alpha}$ is a semi ${ }^{\star}-\mathcal{I}$-closed (resp. semipre se $^{\star}$ $\mathcal{I}$-closed) set.

Theorem 2.2. Let $(X, \tau, \mathcal{I})$ be an ideal space and $A \subset X$. Then the following hold.

(a) $\operatorname{SI} c l(A)=A \cup \operatorname{int}\left(c l^{\star}(A)\right)$. 
(b) $\operatorname{spI} \operatorname{cl}(A)=A \cup \operatorname{int}\left(\operatorname{cl} l^{\star}(\operatorname{int}(A))\right)$.

Proof. The proof follows from Theorem 1.3 and Theorem 3.1 of [5].

Every semi $i^{\star}-\mathcal{I}$-closed set is a semipre ${ }^{\star}-\mathcal{I}$-closed set but not the converse as shown by the following Example 2.3. Theorem 2.4 below shows that the reverse direction is true if the set is semi-I-open. Theorem 2.5 gives a characterization of $t-\mathcal{I}$-sets.

Example 2.3. Consider the ideal space $(X, \tau, \mathcal{I})$ where $X=\{a, b, c, d\}, \tau=\{\emptyset,\{d\},\{a, c\},\{a, c, d\}, X\}$ and $\mathcal{I}=\{\emptyset,\{c\},\{d\},\{c, d\}\}$. If $A=\{a\}$, then $\operatorname{int}\left(c^{\star}(\operatorname{int}(A))\right)=\operatorname{int}\left(c l^{\star}(\emptyset)\right)=\emptyset \subset A$ and so $A$ is semipre ${ }^{\star}-\mathcal{I}-$ closed. $^{*}$ Since int $\left(c l^{\star}(A)\right)=$ $\operatorname{int}\left(c l^{\star}(\{a\})\right)=\operatorname{int}(\{a, b, c\})=\{a, c\} \nsubseteq\{a\}, A$ is not semi $i^{\star}-\mathcal{I}$-closed.

Theorem 2.4. Let $(X, \tau, \mathcal{I})$ be an ideal space and $A \subset X$ be semipre ${ }^{\star}-\mathcal{I}-$ closed. If $A$ is semi-I-open, then $A$ is semi ${ }^{\star}-\mathcal{I}$-closed.

Proof. If $A$ is semi-I-open, then $A \subset c l^{\star}(\operatorname{int}(A))$ and so $c l^{\star}(A) \subset c l^{\star}(\operatorname{int}(A))$. Now $\operatorname{int}\left(c l^{\star}(A)\right) \subset$ $\operatorname{int}\left(\operatorname{cl}^{\star}(\operatorname{int}(A))\right) \subset A$ and so $A$ is semi $i^{\star}-\mathcal{I}$-closed.

Theorem 2.5. Let $(X, \tau, \mathcal{I})$ be an ideal space and $A \subset X$. Then the following are equivalent.

(a) $A$ is a $t-\mathcal{I}-$ set.

(b) $A$ is semi $i^{\star}-\mathcal{I}$-closed.

(c) $A$ is a semipre ${ }^{\star}-\mathcal{I}$-closed $\mathcal{B}_{\mathcal{I}}-$ set.

Proof. Enough to prove that (c) $\Rightarrow$ (a). Suppose $A$ is a semipre ${ }^{\star}-\mathcal{I}$-closed $\mathcal{B}_{\mathcal{I}}$-set. Then $A=U \cap V$ where $U$ is open and $V$ is a $t-\mathcal{I}$-set. Now $\operatorname{int}\left(c l^{\star}(A)\right)=\operatorname{int}\left(c l^{\star}(U \cap V)\right) \subset \operatorname{int}\left(c l^{\star}(U) \cap c l^{\star}(V)\right)=$ $\operatorname{int}\left(c l^{\star}(U)\right) \cap \operatorname{int}\left(c l^{\star}(V)\right)=\operatorname{int}\left(c l^{\star}(U)\right) \cap \operatorname{int}(V)=\operatorname{int}\left(c l^{\star}(U) \cap \operatorname{int}(V)\right) \subset \operatorname{int}\left(c l^{\star}(U \cap \operatorname{int}(V))=\right.$ $\operatorname{int}\left(c l^{\star}(\operatorname{int}(U \cap V))\right)=\operatorname{int}\left(c l^{\star}(\operatorname{int}(A))\right) \subset A$ and $\operatorname{so} \operatorname{int}\left(c l^{\star}(A)\right) \subset \operatorname{int}(A) . \operatorname{But} \operatorname{int}(A) \subset \operatorname{int}\left(c l^{\star}(A)\right)$ and so $\operatorname{int}(A)=\operatorname{int}\left(c l^{\star}(A)\right)$ which implies that $A$ is a $t-\mathcal{I}$-set.

The following Example 2.6 shows that the union of two semi ${ }^{\star}-\mathcal{I}_{\text {closed }}$ (resp. semipre ${ }^{\star}-$ $\mathcal{I}$-closed) set is not a semi $i^{\star}-\mathcal{I}$ closed (resp. semipre ${ }^{\star}-\mathcal{I}$-closed) set.

Example 2.6. Consider the ideal space $(X, \tau, \mathcal{I})$ of Example 2.3. If $A=\{a, c\}$ and $B=\{d\}$, then $\operatorname{int}\left(c l^{\star}(A)\right)=\operatorname{int}\left(c l^{\star}(\{a, c\})\right)=\operatorname{int}(\{a, b, c\})=\{a, c\}=A$ and so $A$ is semi $i^{\star}-\mathcal{I}$-closed and hence semipre $^{\star}-\mathcal{I}$-closed. Also, int $\left(c l^{\star}(B)\right)=\operatorname{int}\left(c l^{\star}(\{d\})\right)=\operatorname{int}(\{d\})=\{d\}=B$. Therefore, $B$ is semi $i^{\star}-I-$ closed and so semipre ${ }^{\star}-I-$ closed. But int $\left(\operatorname{cl}^{\star}(\operatorname{int}(A \cup B))\right)=\operatorname{int}\left(\operatorname{cl}^{\star}(\operatorname{int}(\{a, c, d\}))\right)=$ $\operatorname{int}\left(c l^{\star}(\{a, c, d\})\right)=\operatorname{int}(X)=X \nsubseteq A \cup B$ and so $A \cup B$ is not semipre ${ }^{\star}-\mathcal{I}$-closed and hence $A \cup B$ is not semi $i^{\star}-\mathcal{I}$-closed.

A subset $A$ of an ideal space $(X, \tau, \mathcal{I})$ is said to be a $\mathcal{C}_{\mathcal{I}}$-set [8] if $A=U \cap V$ where $U$ is open and $V$ is a semipre ${ }^{\star}-\mathcal{I}$-closed set. We will denote the family of all $\mathcal{C}_{\mathcal{I}}$-set by $\mathcal{C}_{\mathcal{I}}(X)$. The following Theorem 2.7 gives a characterization of $\mathcal{B}_{\mathcal{I}}$-sets and $\mathcal{C}_{\mathcal{I}}$-sets.

Theorem 2.7. Let $(X, \tau, \mathcal{I})$ be an ideal space and $A$ be a subset of $X$. Then the following hold.

(a) $A$ is a $\mathcal{B}_{\mathcal{I}}-$ set if and only if there exists an open set $U$ such that $A=U \cap S \mathcal{I} c l(A)$.

(b) $A$ is a $\mathcal{C}_{\mathcal{I}}$-set if and only if there exists an open set $U$ such that $A=U \cap \operatorname{sp\mathcal {I}cl}(A)$.

Proof. (a) Suppose $A$ is a $\mathcal{B}_{\mathcal{I}}$-set. Then $A=U \cap V$ where $U$ is open and $V$ is a $t$ - $\mathcal{I}$-set. Since $t-\mathcal{I}$-sets are semi ${ }^{\star}-\mathcal{I}$-closed sets, $S \mathcal{I} c l(V)=V$. Now $A=U \cap A \subset U \cap S \mathcal{I} c l(A) \subset$ $U \cap S \mathcal{I} c l(V)=U \cap V=A$ and so $A=U \cap S \mathcal{I} c l(A)$. Conversely, suppose $A=U \cap S \mathcal{I} c l(A)$ for some 
open set $U$. Since $S \mathcal{I} c l(A)$ is semi $i^{\star}-\mathcal{I}$-closed, $\operatorname{int}\left(\operatorname{cl}^{\star}(S \mathcal{I} c l(A))\right) \subset S \mathcal{I} c l(A)$. Also, $\operatorname{int}(S \mathcal{I} c l(A)) \subset$ $\operatorname{int}\left(c^{\star}(S \mathcal{I} c l(A))\right) \subset S \mathcal{I} c l(A)$ and so $\operatorname{int}(S \mathcal{I} c l(A))=\operatorname{int}\left(c l^{\star}(S \mathcal{I} c l(A))\right)$ which implies that $S \mathcal{I} c l(A)$ is a $t-\mathcal{I}$-set. Therefore, $A$ is a $\mathcal{B}_{\mathcal{I}}$-set.

(b) The proof is similar to that of (a).

A subset $A$ of an ideal space $(X, \tau, \mathcal{I})$ is said to be a $\mathcal{A}_{1 \mathcal{I}}-$ set (resp. $\mathcal{B}_{1 \mathcal{I}}-$ set $[4]\left(\alpha_{\mathcal{I}} M_{1}-\right.$ set $\left.\left.[1]\right)\right)$ if $A=U \cap V$ where $U$ is open (resp. $\alpha-\mathcal{I}$-open ) and $c l^{\star}(\operatorname{int}(V))=X$. We will denote the family of all $\mathcal{B}_{1 \mathcal{I}}$-sets (resp. $\mathcal{A}_{1 \mathcal{I}}$-sets) by $\mathcal{B}_{1 \mathcal{I}}(X)$ (resp. $\mathcal{A}_{1 \mathcal{I}}(X)$ ). Clearly, $\mathcal{A}_{1 \mathcal{I}}(X) \subset \mathcal{B}_{1 \mathcal{I}}(X)$. The following Theorem 2.8 shows that $\mathcal{B}_{1 \mathcal{I}}$-sets and $\mathcal{A}_{1 \mathcal{I}}$-sets are nothing but $\alpha-\mathcal{I}$-open sets.

Theorem 2.8. Let $(X, \tau, \mathcal{I})$ be an ideal space. Then $\mathcal{B}_{1 \mathcal{I}}(X)=\alpha \mathcal{I} O(X)=\mathcal{A}_{1 \mathcal{I}}(X)$.

Proof. Suppose $A \in \mathcal{B}_{1 \mathcal{I}}(X)$. Then $A=U \cap V$ where $U$ is $\alpha-\mathcal{I}$-open and $c l^{\star}(\operatorname{int}(V))=X$. Since $V \subset X=\operatorname{int}\left(c l^{\star}(\operatorname{int}(V))\right), V \in \alpha \mathcal{I} O(X)$. Since $\alpha \mathcal{I} O(X)$ is a topology on $X, A \in \alpha \mathcal{I} O(X)$ and so $\mathcal{B}_{1 \mathcal{I}}(X) \subset \alpha \mathcal{I} O(X)$.

Suppose $A \in \alpha \mathcal{I} O(X)$. Then $A \subset \operatorname{int}\left(c l^{\star}(\operatorname{int}(A))\right)$ and so $A=\operatorname{int}\left(c l^{\star}(\operatorname{int}(A))\right) \cap\left(X-\left(\operatorname{int}\left(\operatorname{cl} l^{\star}(\operatorname{int}(A))\right)-\right.\right.$ $A))=\operatorname{int}\left(c l^{\star}(\operatorname{int}(A))\right) \cap\left(\left(X-\operatorname{int}\left(c l^{\star}(\operatorname{int}(A))\right)\right) \cup A\right) . A l s o, c l^{\star}\left(\operatorname{int}\left(\left(X-\operatorname{int}\left(c l^{\star}(\operatorname{int}(A))\right)\right) \cup A\right)\right) \supset$ $c l^{\star}\left(\operatorname{int}\left(X-\operatorname{int}\left(c l^{\star}(\operatorname{int}(A))\right)\right) \cup \operatorname{int}(A)\right)=c l^{\star}\left(\operatorname{int}\left(X-\operatorname{int}\left(c l^{\star}(\operatorname{int}(A))\right)\right)\right) \cup c l^{\star}(\operatorname{int}(A)) \supset c l^{\star}(\operatorname{int}(X-$ $\left.\left.c l^{\star}(\operatorname{int}(A))\right)\right) \cup c l^{\star}(\operatorname{int}(A)) \supset \operatorname{int}\left(X-c l^{\star}(\operatorname{int}(A))\right) \cup c l^{\star}(\operatorname{int}(A)) \supset \operatorname{int}\left(\left(X-c l^{\star}(\operatorname{int}(A))\right) \cup c l^{\star}(\operatorname{int}(A))\right)=$ $\operatorname{int}(X)=X$. Therefore, $A \in \mathcal{A}_{1 \mathcal{I}}(X)$ which implies that $\alpha \mathcal{I} O(X) \subset \mathcal{A}_{1 \mathcal{I}}(X)$.

Clearly, $\mathcal{A}_{1 \mathcal{I}}(X) \subset \mathcal{B}_{1 \mathcal{I}}(X)$. This completes the proof.

A subset $A$ of an ideal space $(X, \tau, \mathcal{I})$ is said to be an $R \mathcal{I}$-open set [17] if $A=\operatorname{int}\left(c l^{\star}(A)\right)$. We will denote the family of all $R \mathcal{I}$-open sets by $R \mathcal{I} O(X)$. In [17], it is established that $R \mathcal{I} O(X)$ is a base for a topology $\tau_{\mathcal{I}}$ and $\tau_{s} \subset \tau_{\mathcal{I}} \subset \tau$ where $\tau_{s}$ is the semiregularization of $\tau$. The following Theorem 2.9 gives characterizations of pre- $\mathcal{I}$-open sets in terms of $R \mathcal{I}$-open sets.

Theorem 2.9. Let $(X, \tau, \mathcal{I})$ be an ideal space and $A \subset X$. Then the following are equivalent.

(a) $A$ is pre-I-open.

(b) There exists an $R \mathcal{I}$-open set $G$ such that $A \subset G$ and $c l^{\star}(G)=c l^{\star}(A)$.

(c) $A=G \cap D$ where $G$ is $R \mathcal{I}$-open and $D$ is $\tau^{\star}$-dense.

(d) $A=G \cap D$ where $G$ is open and $D$ is $\tau^{\star}$-dense.

Proof. (a) $\Rightarrow(b)$. Suppose $A$ is pre-I-open. If $G=\operatorname{int}\left(c l^{\star}(A)\right)$, then $A \subset G$ and $\operatorname{int}\left(c l^{\star}(G)\right)=$ $\operatorname{int}\left(c l^{\star}\left(\operatorname{int}\left(c l^{\star}(A)\right)\right)\right)=\operatorname{int}\left(c l^{\star}(A)\right)=G$ which implies that $G$ is an $R \mathcal{I}$-open set containing $A$. Also, $c l^{\star}(A) \subset c l^{\star}(G)=c l^{\star}\left(\operatorname{int}\left(c l^{\star}(A)\right)\right) \subset c l^{\star}(A)$ which implies that $c l^{\star}(A)=c l^{\star}(G)$. This proves $(b)$.

$(b) \Rightarrow(c)$. Suppose $G$ is an $R \mathcal{I}$-open set such that $A \subset G$ and $c l^{\star}(G)=c l^{\star}(A)$. If $D=A \cup(X-G)$, then $A=G \cap D$ and $D$ is $\tau^{\star}$-dense. This proves (c).

$(c) \Rightarrow(d)$ is clear.

(d) $\Rightarrow$ (a) follows from Lemma 4.3 of [3].

A subset $A$ of an ideal space $(X, \tau, \mathcal{I})$ is said to be a $\mathcal{A}_{2 \mathcal{I}}-$ set (resp. $\mathcal{B}_{2 \mathcal{I}}-$ set $[4]\left(\alpha_{\mathcal{I}} M_{2}-\right.$ set $\left.[1]\right)$ ) if $A=U \cap V$ where $U$ is open (resp. $\alpha-\mathcal{I}$-open ) and $c l^{\star}(V)=X$. We will denote the family of all $\mathcal{A}_{2 \mathcal{I}}$-sets (resp. $\mathcal{B}_{2 \mathcal{I}}$-sets) by $\mathcal{A}_{2 \mathcal{I}}(X)$ (resp. $\mathcal{B}_{2 \mathcal{I}}(X)$ ). Clearly, $\mathcal{A}_{2 \mathcal{I}}(X) \subset \mathcal{B}_{2 \mathcal{I}}(X)$. The following Theorem 2.10 shows that $\mathcal{A}_{2 \mathcal{I}}$-sets and $\mathcal{B}_{2 \mathcal{I}}$-sets are nothing but pre- $\mathcal{I}$-open sets. Also, it shows that the converse of Proposition 2.6 of [4] is true.

Theorem 2.10. Let $(X, \tau, \mathcal{I})$ be an ideal space. Then $\mathcal{A}_{2 \mathcal{I}}(X)=P \mathcal{I} O(X)=\mathcal{B}_{2 \mathcal{I}}(X)$. 
Proof. By Theorem 2.9(d), $\mathcal{A}_{2 \mathcal{I}}(X)=P \mathcal{I} O(X)$. Since $\mathcal{A}_{2 \mathcal{I}}(X) \subset \mathcal{B}_{2 \mathcal{I}}(X)$, it is enough to prove that $\mathcal{B}_{2 \mathcal{I}}(X) \subset \mathcal{A}_{2 \mathcal{I}}(X)$. Suppose $A \in \mathcal{B}_{2 \mathcal{I}}(X)$. Then $A=U \cap V$ where $U$ is $\alpha-\mathcal{I}$-open and $c l^{\star}(V)=X$. Now $A \subset U \subset \operatorname{int}\left(c l^{\star}(\operatorname{int}(U))\right)=\operatorname{int}\left(c l^{\star}(\operatorname{int}(U \cap X))\right)=\operatorname{int}\left(c l^{\star}\left(\operatorname{int}\left(U \cap c l^{\star}(V)\right)\right)\right) \subset$ $\operatorname{int}\left(c l^{\star}\left(\operatorname{int}\left(c l^{\star}(U \cap V)\right)\right)\right)=\operatorname{int}\left(c l^{\star}(U \cap V)\right)=\operatorname{int}\left(c l^{\star}(A)\right)$ and so $A \in P \mathcal{I} O(X)$. This completes the proof.

Clearly, $A_{1 \mathcal{I}}(X) \subset A_{2 \mathcal{I}}(X)$. The following Example 2.11 shows that an $A_{2 \mathcal{I}}$-set need not be an $A_{1 \mathcal{I}}$-set.

Example 2.11. Consider the ideal space $(X, \tau, \mathcal{I})$ where $X=\{a, b, c\}, \tau=\{\emptyset,\{c\}, X\}$ and $\mathcal{I}=$ $\{\emptyset,\{c\}\}$. If $A=\{a, c\}$, then $A$ is an $A_{2 \mathcal{I}}-$ set. But $c l^{\star}(\operatorname{int}(A))=\operatorname{int}(A) \cup(\operatorname{int}(A))^{\star}=\{c\} \neq X$. Hence $A$ is not an $A_{1 \mathcal{I}}$-set.

A subset $A$ of an ideal space $(X, \tau, \mathcal{I})$ is said to be an $\alpha_{\mathcal{I}} N_{5}$-set [1] if $A=U \cap V$ where $U$ is $\alpha-\mathcal{I}$-open and $V$ is $\star$-closed. We will denote the family of all $\alpha_{\mathcal{I}} N_{5}$-sets of an ideal space $(X, \tau, \mathcal{I})$ by $\alpha_{\mathcal{I}} N_{5}(X)$. A subset $A$ of an ideal space $(X, \tau, \mathcal{I})$ is said to be an $\mathcal{I}$-locally closed [6] (resp. weakly

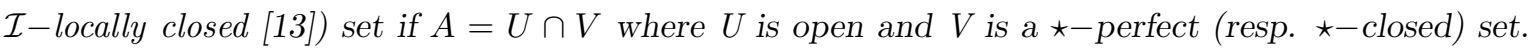
By Theorem 2.9 of [15], $A$ is weakly $\mathcal{I}$-locally closed if and only if $A=U \cap c l^{\star}(A)$ for some open set $U$. The family of all weakly $\mathcal{I}$-locally closed sets is denoted by $W \mathcal{I} L C(X)$. Clearly, every weakly $\mathcal{I}$-locally closed set is an $\alpha_{\mathcal{I}} N_{5}$-set but not the converse as shown by the following Example 2.12. Theorem 2.13 below gives a characterization of $\alpha_{\mathcal{I}} N_{5}-$ sets.

Example 2.12. [4, Example 2.2]Consider the ideal space $(X, \tau, \mathcal{I})$ where $X=\{a, b, c\}$, $\tau=\{\emptyset,\{a\},\{a, c\}, X\}$ and $\mathcal{I}=\{\emptyset,\{b\},\{c\},\{b, c\}\}$. If $A=\{a, b\}$, then $\operatorname{int}\left(c l^{\star}(\operatorname{int}(A))\right)=$ $\operatorname{int}\left(c l^{\star}(\operatorname{int}(\{a, b\}))\right)=\operatorname{int}\left(c l^{\star}(\{a\})\right)=\operatorname{int}(\{a, b, c\})=X \supset A$ and so $A$ is $\alpha-\mathcal{I}$-open and hence an $\alpha_{\mathcal{I}} N_{5}-$ set. But there is no open set $U$ such that $A=U \cap c l^{\star}(A)$ where $c l^{\star}(A)=X$. Hence $A$ is not a weakly $\mathcal{I}$-locally closed set.

Theorem 2.13. Let $(X, \tau, \mathcal{I})$ be an ideal space and $A \subset X$. Then $A$ is $\alpha_{\mathcal{I}} N_{5}-$ set if and only if $A=U \cap c l^{\star}(A)$ for some $U \in \alpha \mathcal{I} O(X)$.

Proof. If $A$ is an $\alpha_{\mathcal{I}} N_{5}$-set, then $A=U \cap V$ where $U$ is $\alpha-\mathcal{I}$-open and $V$ is $\star$-closed. Since $A \subset V, c l^{\star}(A) \subset c l^{\star}(V)=V$ and so $U \cap c l^{\star}(A) \subset U \cap V=A \subset U \cap c l^{\star}(A)$ which implies that $A=U \cap c l^{\star}(A)$. Conversely, suppose $A=U \cap c l^{\star}(A)$ for some $U \in \alpha \mathcal{I} O(X)$. Since $c l^{\star}(A)$ is $\star-$ closed, $A$ is an $\alpha_{\mathcal{I}} N_{5}-$ set.

$A$ subset $A$ of an ideal space $(X, \tau, \mathcal{I})$ is said to be an $\mathcal{I} R-\operatorname{closed}$ set [1] if $A=c l^{\star}(\operatorname{int}(A))$. A subset $A$ of an ideal space $(X, \tau, \mathcal{I})$ is said to be an $\alpha \mathcal{A}_{I}-\operatorname{set}[4]\left(\alpha_{\mathcal{I}} N_{2}-\operatorname{set}[1]\right)\left(\operatorname{resp} . \mathcal{A}_{\mathcal{I} R}-\operatorname{set}[1]\right)$ if $A=U \cap V$ where $U$ is an $\alpha-\mathcal{I}$-open (resp. open) set and $V$ is an $\mathcal{I} R$-closed set. $\mathcal{A}_{\mathcal{I} R}-$ sets are called as $\mathcal{A}_{\mathcal{I}}-$ sets in [4]. We will denote the family of all $\alpha \mathcal{A}_{I}-$ sets (resp. $\mathcal{A}_{\mathcal{I} R}-$ sets) by $\alpha \mathcal{A}_{\mathcal{I}}(X)\left(\operatorname{resp} . \mathcal{A}_{\mathcal{I} R}(X)\right)$. Clearly, every $\mathcal{A}_{\mathcal{I} R}$-set is an $\alpha \mathcal{A}_{I}$-set but the converse is not true [4, Example 2.2]. Theorem 2.14 below shows that $\alpha \mathcal{A}_{I}$-sets are nothing but semi-I- $\mathcal{I}$-open sets which shows that the reverse direction of Proposition 2.4 of [4] is true and each such set is both a strong $\beta-\mathcal{I}$-open set and an $\alpha_{\mathcal{I}} N_{5}$-set.

Theorem 2.14. Let $(X, \tau, \mathcal{I})$ be an ideal space. Then $\alpha \mathcal{A}_{\mathcal{I}}(X)=s \beta \mathcal{I} O(X) \cap \alpha_{\mathcal{I}} N_{5}(X)=S \mathcal{I} O(X)$.

Proof. Suppose $A \in \alpha \mathcal{A}_{\mathcal{I}}(X)$. Then $A=U \cap V$ where $U \in \alpha \mathcal{I} O(X)$ and $V$ is an $\mathcal{I} R$-closed set. Now $A=U \cap V \subset \operatorname{int}\left(c l^{\star}(\operatorname{int}(U))\right) \cap c l^{\star}(\operatorname{int}(V)) \subset c l^{\star}\left(\operatorname{int}\left(c l^{\star}(\operatorname{int}(U))\right) \cap \operatorname{int}(V)\right)=c l^{\star}\left(\operatorname{int}\left(c l^{\star}(\operatorname{int}(U)) \cap\right.\right.$ $\operatorname{int}(V))) \subset c l^{\star}\left(\operatorname{int}\left(c l^{\star}(\operatorname{int}(U) \cap \operatorname{int}(V))\right)\right)=c l^{\star}\left(\operatorname{int}\left(c l^{\star}(\operatorname{int}(U \cap V))\right)\right)=c l^{\star}(\operatorname{int}(U \cap V))=c l^{\star}(\operatorname{int}(A)) \subset$ 


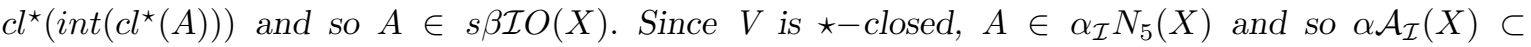
$s \beta \mathcal{I} O(X) \cap \alpha_{\mathcal{I}} N_{5}(X)$. Conversely, suppose $A \in s \beta \mathcal{I} O(X) \cap \alpha_{\mathcal{I}} N_{5}(X) . A \in s \beta \mathcal{I} O(X)$ implies that $A \subset c l^{\star}\left(\operatorname{int}\left(c l^{\star}(A)\right)\right)$ and $A \in \alpha_{\mathcal{I}} N_{5}(X)$ implies that $A=U \cap c l^{\star}(A)$ where $U \in \alpha \mathcal{I} O(X)$. Since $A \subset U, A \subset U \cap c l^{\star}\left(\operatorname{int}\left(c l^{\star}(A)\right)\right) \subset U \cap c l^{\star}(A)=A$ and so $A=U \cap c l^{\star}\left(\operatorname{int}\left(c l^{\star}(A)\right)\right)$. Since $c l^{\star}\left(\operatorname{int}\left(c l^{\star}(A)\right)\right)$ is $\mathcal{I} R$-closed, $A \in \alpha \mathcal{A}_{\mathcal{I}}(X)$ and so $s \beta \mathcal{I} O(X) \cap \alpha_{\mathcal{I}} N_{5}(X) \subset \alpha \mathcal{A}_{\mathcal{I}}(X)$. Therefore, $\alpha \mathcal{A}_{\mathcal{I}}(X)=s \beta \mathcal{I} O(X) \cap \alpha_{\mathcal{I}} N_{5}(X)$.

If $A \in S \mathcal{I O}(X)$, then $A \in s \beta \mathcal{I O}(X)$ by Proposition 1(d) of [9]. Moreover, if $V=A \cup(X-$ $\left.c l^{\star}(\operatorname{int}(A))\right)$, then $A=V \cap c l^{\star}(\operatorname{int}(A))$. Also, $\operatorname{int}\left(c l^{\star}(\operatorname{int}(V))\right)=\operatorname{int}\left(c l^{\star}\left(\operatorname{int}\left(A \cup\left(X-c l^{\star}(\operatorname{int}(A))\right)\right)\right)\right) \supset$ $\operatorname{int}\left(c l^{\star}\left(\operatorname{int}(A) \cup \operatorname{int}\left(X-c l^{\star}(\operatorname{int}(A))\right)\right)\right)=\operatorname{int}\left(c l^{\star}(\operatorname{int}(A)) \cup c l^{\star}\left(\operatorname{int}\left(X-c l^{\star}(\operatorname{int}(A))\right)\right)\right) \supset \operatorname{int}\left(c l^{\star}(\operatorname{int}(A))\right.$ $\left.\cup \operatorname{int}\left(X-c l^{\star}(\operatorname{int}(A))\right)\right) \supset \operatorname{int}\left(\operatorname{int}\left(c l^{\star}(\operatorname{int}(A)) \cup\left(X-c l^{\star}(\operatorname{int}(A))\right)\right)\right)=\operatorname{int}(X)=X \supset V$ and so $V$ is $\alpha$ - I-open. Therefore, $A \in \alpha_{\mathcal{I}} N_{5}(X)$ and hence $S \mathcal{I} O(X) \subset s \beta \mathcal{I} O(X) \cap \alpha_{\mathcal{I}} N_{5}(X)$. Conversely, suppose $A \in s \beta \mathcal{I} O(X) \cap \alpha_{\mathcal{I}} N_{5}(X) . A \in \alpha_{\mathcal{I}} N_{5}(X)$ implies that $A=U \cap V$ where $U$ is

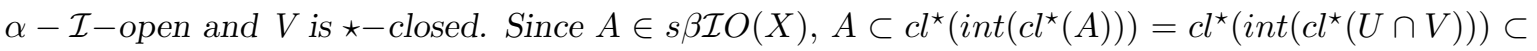
$c l^{\star}\left(\operatorname{int}\left(c l^{\star}\left(\operatorname{int}\left(c l^{\star}(\operatorname{int}(U))\right) \cap V\right)\right)\right) \subset$

$c l^{\star}\left(\operatorname{int}\left(c l^{\star}\left(\operatorname{int}\left(c l^{\star}(\operatorname{int}(U))\right)\right) \cap V\right)\right)=c l^{\star}\left(\operatorname{int}\left(c l^{\star}(\operatorname{int}(U)) \cap V\right)\right)=c l^{\star}\left(\operatorname{int}\left(c l^{\star}(\operatorname{int}(U))\right) \cap \operatorname{int}(V)\right) \subset$ $c l^{\star}\left(\operatorname{int}\left(c l^{\star}(\operatorname{int}(U) \cap \operatorname{int}(V))\right)\right)=c l^{\star}\left(\operatorname{int}\left(c l^{\star}(\operatorname{int}(U \cap V))\right)\right)=c l^{\star}(\operatorname{int}(U \cap V))=c l^{\star}(\operatorname{int}(A))$. Therefore, $A \in S \mathcal{I} O(X)$ which implies that $s \beta \mathcal{I} O(X) \cap \alpha_{\mathcal{I}} N_{5}(X) \subset S \mathcal{I} O(X)$. Hence $s \beta \mathcal{I} O(X) \cap \alpha_{\mathcal{I}} N_{5}(X)=$ $S \mathcal{I O}(X)$.

Corollary 2.15. Let $(X, \tau, \mathcal{I})$ be an ideal space and $A \subset X$. Then the following are equivalent.

(a) $A$ is $\alpha-\mathcal{I}-o p e n$.

(b) $A$ is pre-I-open and semi-I-open [4, Proposition 1.1].

(c) $A$ is a $\mathcal{B}_{2 \mathcal{I}}-$ set and $\alpha \mathcal{A}_{\mathcal{I}}-\operatorname{set}[4$, Theorem 2.3].

Proof. (a) and (b) are equivalent by Proposition 1.1 of [4]. (b) and (c) are equivalent by Theorem 2.10 and Theorem 2.14 .

Corollary 2.16. Let $(X, \tau, \mathcal{I})$ be an ideal space and $A \subset X$. Then the following are equivalent.

(a) $A$ is open.

(b) $A$ is $\alpha-\mathcal{I}$-open and $\mathcal{A}_{\mathcal{I} R}-$ set.

(c) $A$ is pre- $-\mathcal{I}$-open and $\mathcal{A}_{\mathcal{I} R}-$ set.

(d) $A$ is $\alpha-\mathcal{I}$-open and weakly $\mathcal{I}$-locally closed.

(e) $A$ is $\alpha-\mathcal{I}$-open and $\mathcal{B}_{\mathcal{I}}-$ set.

(f) $A$ is $\alpha-\mathcal{I}$-open and $\mathcal{C}_{\mathcal{I}}-$ set.

Proof. (a) and (b) are equivalent by Theorem 2.1 of [4].

That (b) implies (c) is clear.

(c) and (d) are equivalent by Proposition 2.2 of [4].

(d) implies (e) and (e) implies (f) are clear.

(f) implies (a) follows from Proposition 3.3 of [8].

$A$ subset $A$ of an ideal space $(X, \tau, \mathcal{I})$ is said to be a $\mathcal{A}_{3 \mathcal{I}}-$ set (resp. $\mathcal{B}_{3 \mathcal{I}}-$ set $[4]\left(\alpha_{\mathcal{I}} N_{1}-\right.$ set $\left.\left.[1]\right)\right)$

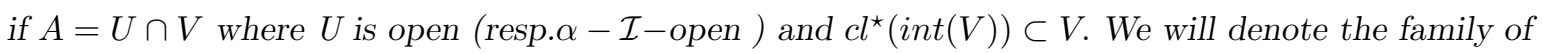
all $\mathcal{A}_{3 \mathcal{I}}$-sets (resp. $\mathcal{B}_{3 \mathcal{I}}$-sets) by $\mathcal{A}_{3 \mathcal{I}}(X)$ (resp. $\mathcal{B}_{3 \mathcal{I}}(X)$ ). Clearly, $\mathcal{A}_{3 \mathcal{I}}(X) \subset \mathcal{B}_{3 \mathcal{I}}(X)$. The following Example 2.17 shows that the reverse direction is not true. Example 2.18 below shows that $A_{2 \mathcal{I}}$-sets and $A_{3 \mathcal{I}}$-sets are independent concepts. Theorem 2.19 below gives a characterization of $\mathcal{A}_{\mathcal{I} R}-$ sets in terms of $\mathcal{A}_{3 \mathcal{I}}$-sets. 
Example 2.17. Consider the ideal space $(X, \tau, \mathcal{I})$ of Example 2.12. If $A=\{a, b\}$, then int $\left(c^{\star}(\operatorname{int}(A))\right)$ $=\operatorname{int}\left(c l^{\star}(\operatorname{int}(\{a, b\}))\right)=\operatorname{int}\left(c l^{\star}(\{a\})\right)=\operatorname{int}(X)=X \supset A$ and so $A$ is an $\alpha-\mathcal{I}$-open set and hence $A$ is a $\mathcal{B}_{3 \mathcal{I}}-$ set. Since $l^{\star}(\operatorname{int}(A)) \nsubseteq A$ and $X$ is the only open set containing $A, A$ is not an $A_{3 \mathcal{I}}-$ set.

Example 2.18. (a) Consider the ideal space $(X, \tau, \mathcal{I})$ of Example 2.12. If $A=\{a, b\}$, then $A$ is not an $A_{3 \mathcal{I}}$-set. Since $c l^{\star}(A)=A \cup A^{\star}=\{a, b\} \cup X=X$, and so $A$ is an $A_{2 \mathcal{I}}-$ set.

(b) Consider the ideal space $(X, \tau, \mathcal{I})$ where $X=\{a, b, c, d\}, \tau=\{\emptyset,\{d\},\{a, c\}$, $\{a, c, d\}, X\}$ and $\mathcal{I}=\{\emptyset,\{c\},\{d\},\{c, d\}\}$. If $A=\{a, b, c\}$, then $c l^{\star}(\operatorname{int}(A))=\operatorname{int}(A) \cup(\operatorname{int}(A))^{\star}=$ $\{a, c\} \cup\{a, b, c\}=\{a, b, c\}=A$ and so $A$ is an $A_{3 \mathcal{I}}-$ set. Since $c l^{\star}(A)=A \cup A^{\star}=\{a, b, c\} \neq X, A$ is not an $A_{2 \mathcal{I}}-$ set.

Theorem 2.19. Let $(X, \tau, \mathcal{I})$ be an ideal space. Then $\mathcal{A}_{\mathcal{I} R}(X)=S \mathcal{I} O(X) \cap \mathcal{A}_{3 \mathcal{I}}(X)$.

Proof. Suppose $A \in \mathcal{A}_{\mathcal{I} R}(X)$. Clearly, $A \in \mathcal{A}_{3 \mathcal{I}}(X)$. By Theorem 3.3 of [1], $A \in S \mathcal{I} O(X)$. Therefore, $\mathcal{A}_{\mathcal{I} R}(X) \subset S \mathcal{I O}(X) \cap \mathcal{A}_{3 \mathcal{I}}(X)$. Conversely, suppose $A \in S \mathcal{I} O(X) \cap \mathcal{A}_{3 \mathcal{I}}(X) . A \in \mathcal{A}_{3 \mathcal{I}}(X)$ implies that $A=U \cap V$ where $U$ is open and $c l^{\star}(\operatorname{int}(V)) \subset V . A \in S \mathcal{I} O(X)$ implies that $A \subset c l^{\star}(\operatorname{int}(A))$ and so $A=A \cap c l^{\star}(\operatorname{int}(A))=(U \cap V) \cap c l^{\star}(\operatorname{int}(U \cap V)) \subset U \cap c l^{\star}(\operatorname{int}(U \cap V))=U \cap c l^{\star}(U \cap \operatorname{int}(V)) \subset$ $U \cap c l^{\star}(U) \cap c l^{\star}(\operatorname{int}(V)) \subset U \cap V=A$ and so $A=U \cap c l^{\star}(\operatorname{int}(U \cap V))=U \cap c l^{\star}(\operatorname{int}(A))$. Since $c l^{\star}(\operatorname{int}(A))$ is $\mathcal{I} R$-closed, $A \in \mathcal{A}_{\mathcal{I} R}(X)$. Therefore, $\mathcal{A}_{\mathcal{I} R}(X)=S \mathcal{I} O(X) \cap \mathcal{A}_{3 \mathcal{I}}(X)$.

Corollary 2.20. Let $(X, \tau, \mathcal{I})$ be an ideal space and $A \subset X$. Then the following are equivalent.

(a) $A \in \mathcal{A}_{\mathcal{I} R}(X)$.

(b) $A \in S \mathcal{I} O(X) \cap \mathcal{A}_{3 \mathcal{I}}(X)$.

(c) $A \in \alpha \mathcal{A}_{\mathcal{I}}(X) \cap \mathcal{A}_{3 \mathcal{I}}(X)$.

(d) $A \in s \beta \mathcal{I} O(X) \cap \alpha_{\mathcal{I}} N_{5}(X) \cap \mathcal{A}_{3 \mathcal{I}}(X)$.

(e) $A \in s \beta \mathcal{I} O(X) \cap W \mathcal{I} L C(X)$.

Proof. (a), (b), (c) and (d) are equivalent by Theorem 2.14 and Theorem 2.19.

(a) and (e) are equivalent by Theorem 2.10 of [15].

By Remark 3.3 of [8], every $\mathcal{B}_{\mathcal{I}}$-set is a $\mathcal{C}_{\mathcal{I}}$-set but the reverse direction is not true. The following Theorem 2.22 gives characterizations of $\mathcal{B}_{\mathcal{I}}$-sets in terms of $\mathcal{C}_{\mathcal{I}}$-sets. A subset $A$ of an ideal space $(X, \tau, \mathcal{I})$ is said to be an $\alpha \mathcal{B}_{\mathcal{I}}-$ set $\left(\alpha_{\mathcal{I}} N_{3}\right.$-set [1]) if $A=U \cap V$ where $U \in \alpha \mathcal{I} O(X)$ and $V$ is a $t-\mathcal{I}$-set. $A$ subset $A$ of an ideal space $(X, \tau, \mathcal{I})$ is said to be an $\alpha \mathcal{C}_{\mathcal{I}}-$ set [4] $\left(\alpha_{\mathcal{I}} N_{4}-\right.$ set [1]) if $A=U \cap V$ where $U \in \alpha \mathcal{I} O(X)$ and $V$ is a $\alpha^{\star}-\mathcal{I}$-set. Clearly every $\alpha \mathcal{B}_{\mathcal{I}}$-set is an $\alpha \mathcal{C}_{\mathcal{I}}-$ set [1, Proposition 3.2(c)] but not the converse [1, Example 3.4]. We will denote the family of all $\alpha \mathcal{B}_{\mathcal{I}}$-sets (resp. $\alpha \mathcal{C}_{\mathcal{I}}-$ sets) in $(X, \tau, \mathcal{I})$ by $\alpha \mathcal{B}_{\mathcal{I}}(X)\left(\operatorname{resp.} \alpha \mathcal{C}_{\mathcal{I}}(X)\right)$. We define $\mathcal{D}_{\mathcal{I}}(X)=\{A \subset X \mid \operatorname{int}(A)=\operatorname{pI} \operatorname{int}(A)\}$ and if $A \in \mathcal{D}_{\mathcal{I}}$, then $A$ is called a $\mathcal{D}_{\mathcal{I}}$-set. The following Lemma 2.21 characterizes $\alpha \mathcal{B}_{\mathcal{I}}$-sets and $\alpha \mathcal{C}_{\mathcal{I}}$-sets, the proof, which is similar to the proof of Theorem 2.7, is omitted. Corollary 2.23 follows from Theorem 2.22.

Lemma 2.21. Let $(X, \tau, \mathcal{I})$ be an ideal space and $A$ be a subset of $X$. Then the following hold.

(a) $A$ is a $\alpha \mathcal{B}_{\mathcal{I}}$-set if and only if there exists an $\alpha-\mathcal{I}$-open set $U$ such that $A=U \cap S \mathcal{I} c l(A)$.

(b) $A$ is an $\alpha \mathcal{C}_{\mathcal{I}}$-set if and only if there exists an $\alpha-\mathcal{I}$-open set $U$ such that $A=U \cap \operatorname{sp} \mathcal{I} c l(A)$.

Theorem 2.22. Let $(X, \tau, \mathcal{I})$ be an ideal space and $A \subset X$. Then the following are equivalent.

(a) $A$ is a $\mathcal{D}_{\mathcal{I}}-$ set and $a \mathcal{C}_{\mathcal{I}}-$ set.

(b) $A$ is a $\delta-\mathcal{I}$-open set and a $\mathcal{C}_{\mathcal{I}}-$ set. 
(c) $A$ is a $\mathcal{B}_{\mathcal{I}}-$ set.

(d) $A$ is an $\alpha \mathcal{B}_{\mathcal{I}}-$ set and a $\mathcal{C}_{\mathcal{I}}-$ set.

Proof. (a) $\Rightarrow(b)$. Suppose $A \in \mathcal{D}_{\mathcal{I}}(X) \cap \mathcal{C}_{\mathcal{I}}(X)$. If $A \in \mathcal{D}_{\mathcal{I}}(X)$, then $\operatorname{int}(A)=\operatorname{pI} \operatorname{int}(A)$. Now $\operatorname{int}\left(c l^{\star}(A)\right)=c l^{\star}(A) \cap \operatorname{int}\left(c l^{\star}(A)\right) \subset c l^{\star}\left(A \cap \operatorname{int}\left(c l^{\star}(A)\right)\right)=c l^{\star}$ $(p \operatorname{Iint}(A))=c l^{\star}(\operatorname{int}(A))$ and so $A$ is a $\delta-\mathcal{I}$-open set. This proves (b).

$(b) \Rightarrow(c)$. Suppose $A$ is a $\delta-\mathcal{I}$-open set and a $\mathcal{C}_{\mathcal{I}}$-set. Then, by Theorem 2.4 of $[12], \operatorname{int}\left(c l^{\star}(A)\right)=$

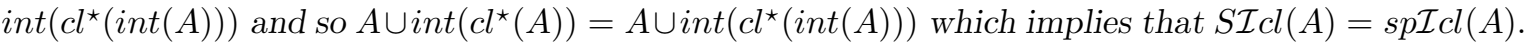
If $A$ is a $\mathcal{C}_{\mathcal{I}}$-set, then Theorem 2.7, $A=U \cap \operatorname{spI} \operatorname{Icl}(A)$ for some open set $U$ and so $A=U \cap S \mathcal{I} c l(A)$ for some open set $U$ which implies that $A$ is a $\mathcal{B}_{\mathcal{I}}$-set.

(c) $\Rightarrow$ (a). Clearly, every $\mathcal{B}_{\mathcal{I}}$-set is a $\mathcal{C}_{\mathcal{I}}$-set. If $A$ is a $\mathcal{B}_{\mathcal{I}}$-set, then $A=U \cap V$ where $U$ is open and $\operatorname{int}\left(c l^{\star}(V)\right)=\operatorname{int}(V)$. Now $p \mathcal{I} \operatorname{int}(A)=A \cap \operatorname{int}\left(c l^{\star}(A)\right)=A \cap \operatorname{int}\left(c l^{\star}(U \cap V)\right) \subset A \cap \operatorname{int}\left(c l^{\star}(U) \cap\right.$ $\left.c l^{\star}(V)\right)=A \cap \operatorname{int}\left(c l^{\star}(U)\right) \cap \operatorname{int}\left(c l^{\star}(V)\right)=(U \cap V) \cap \operatorname{int}\left(c l^{\star}(U)\right) \cap \operatorname{int}(V)=U \cap \operatorname{int}(V)=\operatorname{int}(U \cap V)=$ $\operatorname{int}(A)$. But always, $\operatorname{int}(A) \subset p \mathcal{I} \operatorname{int}(A)$ and so $\operatorname{int}(A)=p \mathcal{I} \operatorname{int}(A)$ which implies that $A$ is a $\mathcal{D}_{\mathcal{I}}-$ set. This proves (a).

$(c) \Rightarrow(d)$ is clear.

$(d) \Rightarrow(c)$. If $A$ is an $\alpha \mathcal{B}_{\mathcal{I}}$-set, then $A=U \cap V$ where $U$ is $\alpha-\mathcal{I}$-open and $\operatorname{int}\left(c l^{\star}(V)\right)=\operatorname{int}(V)$. Now $A \subset U$ implies that $A \subset \operatorname{int}\left(c l^{\star}(\operatorname{int}(U))\right)$ and $\operatorname{so} \operatorname{int}\left(c l^{\star}(A)\right) \subset \operatorname{int}\left(c l^{\star}\left(\operatorname{int}\left(c l^{\star}(\operatorname{int}(U))\right)\right)\right)=$ $\operatorname{int}\left(c l^{\star}(\operatorname{int}(U))\right) \subset \operatorname{int}\left(c l^{\star}(U)\right)$. Again, $A \subset V$ implies that $\operatorname{int}\left(c l^{\star}(A)\right) \subset \operatorname{int}\left(c l^{\star}(V)\right)=\operatorname{int}(V)$. Therefore,

$\operatorname{int}\left(c l^{\star}(A)\right) \subset \operatorname{int}\left(c l^{\star}(U)\right) \cap \operatorname{int}(V) \subset c l^{\star}(\operatorname{int}(U) \cap \operatorname{int}(V)) \subset c l^{\star}(\operatorname{int}(U \cap V))=c l^{\star}(\operatorname{int}(A))$ and so $\operatorname{int}\left(c l^{\star}(A)\right)=\operatorname{int}\left(c l^{\star}(\operatorname{int}(A))\right)$ which implies that $A \cup \operatorname{int}\left(c l^{\star}(A)\right)$

$=A \cup \operatorname{int}\left(c^{\star}(\operatorname{int}(A))\right)$. Hence $\operatorname{SIcl}(A)=\operatorname{sp\mathcal {I}cl}(A)$. Since $A$ is a $\mathcal{C}_{\mathcal{I}}-$ set, by Theorem 2.7, $A=$ $G \cap \operatorname{spI} \operatorname{Icl}(A)$ for some open set $G$ and so $A=G \cap S \mathcal{I} c l(A)$. Therefore, $A$ is a $\mathcal{B}_{\mathcal{I}}$-set.

Corollary 2.23. Let $(X, \tau, \mathcal{I})$ be an ideal space. Then the following hold.

(a) Every $\mathcal{B}_{\mathcal{I}}-$ set is a $\mathcal{D}_{\mathcal{I}}-$ set.

(b) Every $\mathcal{B}_{\mathcal{I}}$-set is a $\alpha \mathcal{B}_{\mathcal{I}}$-set.

(c) Every $\mathcal{D}_{\mathcal{I}}$-set is a $\delta$ - I-open set (Proof follows from $(a) \Rightarrow(b)$ of Theorem 2.22).

The following Theorem 2.24 characterizes $\alpha \mathcal{B}_{\mathcal{I}}$-open sets in terms of $\delta$ - $\mathcal{I}$-open sets and $\alpha \mathcal{C}_{\mathcal{I}}$-open sets. Example 2.25 below shows that $\delta-\mathcal{I}$-openness and $\alpha \mathcal{C}_{\mathcal{I}}$-openness are independent concepts.

Theorem 2.24. Let $(X, \tau, \mathcal{I})$ be an ideal space. Then $\alpha \mathcal{B}_{\mathcal{I}}(X)=\delta \mathcal{I} O(X) \cap \alpha \mathcal{C}_{\mathcal{I}}(X)$.

Proof. Clearly, $\alpha \mathcal{B}_{\mathcal{I}}(X) \subset \alpha \mathcal{C}_{\mathcal{I}}(X)$. If $A \in \alpha \mathcal{B}_{\mathcal{I}}(X)$, then $A=U \cap V$ where $U$ is $\alpha-\mathcal{I}$-open and $V$ is a $t-\mathcal{I}$-set. $A \subset U$ implies that $\operatorname{int}\left(c l^{\star}(A)\right) \subset \operatorname{int}\left(c l^{\star}(U)\right) \subset \operatorname{int}\left(c l^{\star}\left(\operatorname{int}\left(c l^{\star}(\operatorname{int}(U))\right)\right)\right) \subset$ $\operatorname{int}\left(c l^{\star}(\operatorname{int}(U))\right) \subset c l^{\star}(\operatorname{int}(U))$. Also, $A \subset V \operatorname{implies}$ that $\operatorname{int}\left(c l^{\star}(A)\right) \subset \operatorname{int}\left(c l^{\star}(V)\right)=\operatorname{int}(V)$ and so $\operatorname{int}\left(c l^{\star}(A)\right) \subset c l^{\star}(\operatorname{int}(U)) \cap \operatorname{int}(V) \subset c l^{\star}(\operatorname{int}(U) \cap \operatorname{int}(V))=c l^{\star}(\operatorname{int}(U \cap V))=c l^{\star}(\operatorname{int}(A)$. Therefore, $A \in \delta \mathcal{I} O(X)$. Hence $\alpha \mathcal{B}_{\mathcal{I}}(X) \subset \delta \mathcal{I} O(X) \cap \alpha \mathcal{C}_{\mathcal{I}}(X)$. Conversely, suppose $A \in \delta \mathcal{I} O(X) \cap \alpha \mathcal{C}_{\mathcal{I}}(X)$. $A \in \delta \mathcal{I} O(X)$ implies that $\operatorname{int}\left(c l^{\star}(A)\right)=\operatorname{int}\left(c l^{\star}(\operatorname{int}(A))\right)$ and so $\operatorname{SI} \operatorname{Icl}(A)=\operatorname{spI} \mathcal{I} c l(A) . A \in \alpha \mathcal{C}_{\mathcal{I}}(X)$ implies that $A=U \cap \operatorname{spI} c l(A)$ for some $\alpha-\mathcal{I}$-open set $U$ by Lemma 2.21 and so $A=U \cap S \mathcal{I}_{c l}(A)$ for some $\alpha-\mathcal{I}$-open set $U$ which implies that $A \in \alpha \mathcal{B}_{\mathcal{I}}(X)$. Therefore, $\delta \mathcal{I} O(X) \cap \alpha \mathcal{C}_{\mathcal{I}}(X) \subset \alpha \mathcal{B}_{\mathcal{I}}(X)$. This completes the proof.

Example 2.25. (a) Let $X=\{a, b, c, d\}, \tau=\{\emptyset,\{d\},\{a, b\},\{a, b, d\}, X\}$ and $\mathcal{I}=\{\emptyset,\{c\}\}$. If $A=\{a, c\}$, then $\operatorname{int}\left(c l^{\star}(\operatorname{int}(A))\right)=\operatorname{int}\left(c l^{\star}(\operatorname{int}(\{a, c\}))\right)=\operatorname{int}\left(c l^{\star}(\emptyset)\right)=\emptyset=\operatorname{int}(A)$. Therefore, $A$ is 
an $\alpha^{\star}-\mathcal{I}$-set and hence an $\alpha \mathcal{C}_{\mathcal{I}}$-set. But $\operatorname{int}\left(c l^{\star}(A)\right)=\operatorname{int}(\{a, b, c\})=\{a, b\}$ and $c l^{\star}(\operatorname{int}(A))=$ $c l^{\star}(\emptyset)=\emptyset$ and so $A$ is not a $\delta-\mathcal{I}$-set.

(b) Let $X=\{a, b, c, d\}, \tau=\{\emptyset,\{a\},\{c\},\{a, c\}, X\}$ and $\mathcal{I}=\{\emptyset,\{a\}\}$. If $A=\{a, b, c\}$, then $A$ is neither open nor an $\alpha^{\star}-\mathcal{I}$-set and so $A$ is not an $\alpha \mathcal{C}_{\mathcal{I}}$-set. But $\operatorname{int}\left(c l^{\star}(A)\right)=\operatorname{int}(\{a, b, c, d\})=X$ and $c l^{\star}(\operatorname{int}(A))=c l^{\star}(\{a, c\})=X$ and so $A$ is a $\delta-\mathcal{I}$-set.

An ideal space $(X, \tau, \mathcal{I})$ is said to be $\star$-extremally disconnected [7] if the $\tau^{\star}$-closure ( $\star$-closure) of every open set is open. Clearly, $\mathcal{B}_{3 \mathcal{I}}(X) \subset \alpha \mathcal{C}_{\mathcal{I}}(X)$. By Example 3.6 of [1] the reverse direction is not true. The following Theorem 2.26 shows that for $\star$-extremally disconnected spaces, the two collection of sets coincide. Example 2.27 below shows that $\alpha C_{\mathcal{I}}(X)=B_{3 \mathcal{I}}(X)$ does not imply that the space is $\star-$ extremally disconnected.

Theorem 2.26. Let $(X, \tau, \mathcal{I})$ be $a \star$-extremally disconnected ideal space. Then $\mathcal{B}_{3 \mathcal{I}}(X)=\alpha \mathcal{C}_{\mathcal{I}}(X)$.

Proof. Enough to prove that $\alpha \mathcal{C}_{\mathcal{I}}(X) \subset \mathcal{B}_{3 \mathcal{I}}(X)$. Suppose $A \in \alpha \mathcal{C}_{\mathcal{I}}(X)$. Then $A=U \cap V$ where $U$ is $\alpha-\mathcal{I}$-open and $\operatorname{int}\left(c l^{\star}(\operatorname{int}(V))\right)=\operatorname{int}(V)$. Since $(X, \tau, \mathcal{I})$ is $\star$-extremally disconnected, $c l^{\star}(\operatorname{int}(V))$ is open and so $\operatorname{int}(V)=\operatorname{int}\left(c l^{\star}(\operatorname{int}(V))\right)=c l^{\star}(\operatorname{int}(V))$. Therefore, $A \in \mathcal{B}_{3 \mathcal{I}}(X)$. This completes the proof.

Example 2.27. Consider the ideal space $(X, \tau, \mathcal{I})$ where $X=\{a, b, c\}, \tau=\{\emptyset,\{b\},\{c\},\{b, c\}, X\}$ and $\mathcal{I}=\{\emptyset,\{a\}\}$. If $A=\{b\}, A$ is open and $c l^{\star}(A)=\{b\} \cup\{a, b\}=\{a, b\}$, which is not open. Hence $(X, \tau, \mathcal{I})$ is not $\star$-extremally disconnected but $\wp(X)=\alpha C_{\mathcal{I}}(X)=B_{3 \mathcal{I}}(X)$.

Received: November 2008. Revised: February 2009.

\section{References}

[1] A. AÇIKGÖz And Ş.Yüksel, Some new sets and Decompositions of $A_{\mathcal{I}-R}$-continuity, $\alpha-$ $\mathcal{I}$-continuity, Continuity via Idealization, Acta Math. Hungar., 114(1 - 2)(2007), 79 - 89.

[2] A. AÇIKGÖz, T. NoIRI And Ş.YÜKsel, On $\delta$ - I-open sets and Decomposition of $\alpha$-continuity, Acta Math. Hungar., 102(2004), 349 - 357.

[3] A. AÇIKGÖz, Ş.YÜKSEL AND NoIRI, $\alpha-\mathcal{I}$-Preirresolute Functions and $\beta-\mathcal{I}$-Preirresolute Functions, Bull. Malays. Math. Sci. Soc., (2)28(1)(2005), 1 - 8.

[4] G. Aslim, A. Caksu Guler and T. Noiri, On decompositions of continuity and some weaker forms of continuity via idealization, Acta Math. Hungar., 109(3)(2005), 183 - 190.

[5] Á. Császár, On the $\gamma$-interior and $\gamma$-closure of a set, Acta Math. Hungar., 80(1998), 89 - 93.

[6] J. Dontchev, On pre-I- -open sets and a decomposition of $\mathcal{I}$-continuity, Banyan Math.J., 2(1996).

[7] E. EKICI AND T. NoIRI, «-extremally disconnected ideal topological spaces, Acta Math. Hungar., to appear.

[8] E. Hatir And T. Noiri, On decomposition of Continuity via Idealization, Acta Math. Hungar., 96(4)(2002), $341-349$. 
[9] E. Hatir, A. Keskin And T. Noiri, On a new decomposition of continuity via idealization, JP Jour. Geometry and Topology, 3(2003), 53 - 64.

[10] E. Hayashi, Topologies defined by local properties, Math. Ann., 156(1964), 205 - 215.

[11] D. Jankovic And T.R. Hamlett, New Topologies from old via Ideals, Amer. Math. Monthly, 97 No.4 (1990), 295 - 310.

[12] V. Jeyanthi and D. Sivaraj, A note on $\delta$ - I-sets, Acta Math. Hungar., 114(1 - 2)(2007), $165-169$.

[13] A. Keskin, Ş. Yüksel and T. NolRI, Decompositions of $\mathcal{I}$-continuity and Continuity, Commun. Fac. Sci. Univ. Ank. Serials A1, 53(2004), 67 - 75.

[14] K. Kuratowski, Topology, Vol. I, Academic Press, New York, 1966.

[15] V. Renukadevi, Note on $\mathcal{I} R$-closed and $A_{\mathcal{I} R}-$ sets, Acta Math. Hungar., To appear.

[16] R. Vaidyanathaswamy, The Localization Theory in Set Topology, Proc. Indian Acad. Sci. Math. Sci., 20 (1945), 51 - 61.

[17] S. Yüksel, A. AÇIKGÖZ And T. NoIRI, On $\delta$ - I-continuous functions, Turk. J. Math., 29(2005), $39-51$. 